\title{
Intradimensional and extradimensional shifts in compound concept learning
}

\author{
MAY F. D'AMATO AND RICHARD RYAM
}

BROOKLYN COLLEGE

Following pretraining in a two category concept task with one of two dimensions relevant, Ss were shifted to a second concept task requiring four response categories with two of three dimensions relevant. Training on the second (compound) concept involved either an intradimensional, an extradimensional, or a control shift relative to pretraining. Compound concept learning requiring an IS was shown to be superior to learning requiring an $\mathrm{ES}$, and to yield positive transfer. Compound concept learning requiring an ES did not produce negative transfer.

A number of studies with human adults involving the learning of two successive concepts (e.g., Harrow, 1964; Uhl, 1966) have shown that when first and second concepts have the same relevant dimensions-intradimensional shifts (ISs)-performance is better than when relevant and irrelevant dimensions are switched between the two concepts-extradimensional shifts (ESs). The standard procedure in these studies has involved one relevant dimension for both first and second concept learning. Similar results would be expected if the second concept were to involve, in addition, a new relevant dimension. That is, if the second concepts were compound concepts requiring $\mathrm{S}$ to respond to a new, additional dimension, and also make a shift relative to pretraining with the first concept, then those compound concepts requiring an IS should be learned faster than those requiring an ES. This would be expected since pretraining would serve to strengthen mediating responses appropriate to the relevant dimension of the first concept. When the compound concept requires an IS, $S$ will then have half the number of necessary mediators readily available. For this reason, Ss learning compound concepts requiring an IS should also show positive transfer when compared to control Ss pretrained with stimuli unrelated to those used for the compound concept. In addition, assuming that mediating responses do not extinguish very rapidly, negative transfer may be expected in compound concept learning requiring an ES, since at the outset of such learning the dominant mediators will be inappropriate. The present study seeks to test these predictions stemming from mediation theory.

Method

Subjects. A total of 90 Brooklyn College undergraduates served individually as Ss. Of these, two were eliminated for inability to solve the first (pretraining) concept, one for inability to solve the second (training) concept, and 15 due to procedural errors.
These 15 came from all experimental groups, whereas the non-solvers were in size relevant groups. The non-solver of the second concept had received an ES. Six men and six women, otherwise randomly assigned, were placed in each of the six groups involved in the study.

Apparatus. The stimuli consisted of forms pasted on $3 \times 5$ oak tag cards and presented manually through a $3-1 / 4 \times 3$ in. opening in a $20 \times 40$ in. black screen. The screen was on a table, with $S$ seated $3-1 / 2 \mathrm{ft}$. in front of it, and $E$ behind it. The same deck of four stimulus cards was used for the experimental groups during pretraining. It consisted of a square, centered on each card, which varied in size (14/16 or $17 / 16$ in.) and shade (Color-Vu gray 7 or 11). The deck used for pretraining with the control groups also consisted of four cards, but each contained an upright red semicircle $1 \mathrm{in}$. in diameter. This form varied in location $(1 / 2$ in. off center to the right or left) and orientation (diameter facing center or outside of card). During training the same deck of eight cards was used for all groups. It consisted of the same shade and size combinations used for pretraining with the experimental groups, with a third dimension being addedpresence or absence of a 1/4-in. white dot in the center of each square.

Procedure. Prior to pretraining each $\mathrm{S}$ was informed that he would be shown a series of cards, one at a time, each of which he was to verbally identify as a "Koj" or "Mec," and that all cards bearing the same name had something in common. The correct response was orally presented by $\mathrm{E}$, either immediately after $S$ made his choice or after 5 sec. if $S$ had made no response. The stimuli were presented in eight random orders of the four possible stimulus patterns with the restrictions that the same card could not appear twice in a row nor could the same correct response occur more than three times in a row. Each $S$ received a different sequence of this type. For half the experimental Ss, the relevant dimension was size with shade irrelevant, while for the remaining half shade was relevant with size irrelevant. All control Ss had orientation relevant with location irrelevant. The specific response assigned to a value of a relevant dimension was randomly varied. The criterion used was 15 successive correct responses, and Ss failing to reach this in 128 trials were discarded. Following pretraining Ss were asked to defne "Koj" and "Mec," which all but one did successfully, and were then instructed in the training problem. The 
procedure for training was basically the same as for pretraining. However, since training involved two relevant dimensions, each with two values, the responses "L," "R," "U," and "Z"' were requird. The presence or absence of the dot was one of the two relevant dimensions for all Ss. For half the Ss, size was the second relevant dimension, while for the remaining Ss it was shade. Thus, the six groups of the study included three learning the compound concept with shade relevant, and three with size relevant. of the three groups learning each compound concept one was required to make an IS, another to make an ES, and the third served as a control group. A maximum of 160 trials was allowed.

\section{Results and Discussion}

All results are in terms of the number of trials to concept attainment excluding the 15 criterion trials. A $\sqrt{\mathrm{X}+.5}$ transformation applied to the pretraining data reduced, but did not eliminate, heterogeneity of variance. A 2 by 3 by 2 analysis of variance involving sex, type of shift to be required (IS, ES, or control), and training concept to be learned (size or shade as one of the two relevant dimensions) was performed on the transformed pretraining data. The only significant source of variance was an interaction between type of shift and training concept, $F=4.28, \mathrm{df}=2 / 60$, $p<.05$. The pattern of means suggested that the interaction reflected differences in the difficulty of the pretraining concepts, size being most difficult, orientation next, and shade least difficult. However, Scheffé tests revealed none of the differences in difficulty to be significant.

Training data showed heterogeneity of variance which was eliminated by a $\sqrt{\mathrm{X}+.5}$ transformation. A 2 by 3 by 2 analysis of variance was carried out on these transformed data to evaluate the effects of sex, type of shift, and training concept. The analysis revealed a significant main effect for type of shift, $\mathrm{F}=8.10, \mathrm{df}=2 / 60, \mathrm{p}<.01$ Specific comparisons were made using the Scheffe' test which required a difference between means to be equal to or greater than .98 for significance at the .05 level. The means for each type of shift were: IS, 3.48; ES, 4.98; Control, 4.68. (The raw score means for each type of shift were: IS, 13.58; ES, 25.67; Control, 23.92.) The only other significant source of variance emerging from the analysis stemmed from the greater difficulty of the compound concept involving size as compared to shade, $\mathrm{F}=4.48$, $\mathrm{df}=1 / 60, \mathrm{p}<.05$.

Compound concept learning requiring an IS has been shown in this study to be superior to compound concept learning requiring an ES and, further, to yield positive transfer. Thus, these results are consistent with expectations stemming from mediation theory. Similar findings have been obtained by Petre (1964). Petre, however, used only one and the same compound concept for all Ss, with each experimental group required to make only one type of shift. Due to the resulting confounding between type of shift and type of pretraining, facilitation in compound concept learning requiring an IS was not clearly demonstrated in his study.

The results of the present study also showed that concept learning requiring an ES did not result in negative transfer. In some other studies also failing to obtain negative transfer, control groups were given no pretraining (e.g., Kendler \& D'Amato, 1955, Experiment III; Petre, 1964). Without pretrained control groups, the absence of negative transfer following an $E S$, as well as the presence of positive transfer following an IS, may be attributed, to some extent at least, to the failure of control groups to benefit from nonspecific transfer. The absence of negative transfer in the present study may have been due to the relatively rapid extinction of inappropriate mediating responses when an ES was required.

\section{References}

Harrow, M. Stimulus aspects responsible for the rapid acquisition of reversal shifts in concept formation. J. exp. Psychol., 1964 $67,330-334$.

Kendler, H. H., \& D'Amato, M. F. A comparison of reversal shifts and nonreversal shifts in human concept formation behavior. J. exp. Psychol., 1955, 49, 165-174.

Petre, R. D. Concept acquisition as a function of stimulus-equivalence pretraining with identical and dissimilar stimuli. $J$. exp. Psychol., 1964, 67, 360-364.

Uhl, $\mathbf{N}$. Intradimensional and extradimensional shifts as a function of amount of training and similarity between training and shift stimuli. J. exp. Psychol., 1966, 72, 429-433. 